\title{
Análise da qualidade de adesão de diferentes bases de braquetes metálicos
}

\author{
Danielle Morello Park*, Fábio Lourenço Romano**, Ary dos Santos-Pinto***, Lídia Parsekian Martins ${ }^{\star \star \star \star}$, Darcy \\ Flávio Nouer ${ }^{\star \star \star \star \star}$
}

\begin{abstract}
Resumo
Diante das diferenças existentes nas características das bases dos braquetes usados atualmente, objetivou-se neste trabalho comparar entre si três tipos de bases de braquetes metálicos (Monobloc, Equilibrium e Dynalock). Foram utilizados 36 pré-molares humanos, divididos em 3 grupos de 12 dentes. Os dentes foram incluídos em troquéis com gesso pedra tipo IV e posicionados com suas faces vestibulares perpendiculares à base do troquel. Todos os braquetes foram colados com o compósito Concise Ortodôntico e submetidos ao ensaio de cisalhamento em uma Máquina Universal com uma velocidade de $0,5 \mathrm{~mm}$ por minuto. $\mathrm{O}$ braquete Monobloc obteve o maior valor médio de resistência adesiva $\left(\mathrm{x}=28,19 \mathrm{Kgf} / \mathrm{cm}^{2}\right)$, sendo superior estatisticamente aos braquetes Equilibrium ( $\left.\mathrm{x}=18,07 \mathrm{Kgf} / \mathrm{cm}^{2}\right)$ e Dynalock ( $\left.\mathrm{x}=18,24 \mathrm{Kgf} / \mathrm{cm}^{2}\right)$. Em relação ao ARI (Índice de Remanescente Resinoso), não foi encontrada diferença estatística entre os braquetes testados.
\end{abstract}

Palavras-chave: Ortodontia. Colagem. Resistência ao cisalhamento. Braquetes. Base.

\section{INTRODUÇÃO}

A avaliação dos materiais de colagem é extremamente importante para verificação da qualidade de adesão ao esmalte, porém, o tipo de braquete, principalmente as características estruturais de sua base, assim como o número e forma de suas retenções também devem ser analisados.

Retief e Sadowsky ${ }^{14}$ relataram que forças no sentido ocluso-gengival, ou seja, forças de cisalhamento, são as que mais incidem sobre os acessó- rios ortodônticos, exigindo adequada adesão ao esmalte para que não ocorram falhas clínicas.

Segundo Santos Pinto et al. ${ }^{19}$, a queda de acessórios ortodônticos acontece devido a falhas na técnica de colagem, pouca retentividade de determinadas bases de braquetes e ação da força mastigatória.

Com o intuito de melhorar a resistência adesiva do acessório ortodôntico ao dente, diversos tipos de base de braquetes têm sido testadas $2,4,5,8,9,10,13,20,21$.

* Faculdade de Odontologia de Araraquara/ UNESP; Especialista em Ortodontia e Ortopedia Facial; Aluna do Curso de

Mestrado em Ortodontia.

Faculdade de Odontologia de Piracicaba/ UNICAMP; Especialista em Ortodontia e Ortopedia Facial; Aluno do Curso de Mestrado em Ortodontia.

Faculdade de Odontologia de Araraquara/ UNESP; Professor Adjunto do Departamento de Clínica Infantil; Professor Livre Docente.

* Faculdade de Odontologia de Araraquara/ UNESP; Professora Assistente Doutor do Departamento de Clínica Infantil; Professora Doutora.

Faculdade de Odontologia de Piracicaba/ UNICAMP; Professor responsável pela área de Ortodontia da FOP / UNICAMP; Professor Titular. 
Um ponto a ser salientado é a influência da caracterização estrutural e morfológica da base dos braquetes nos valores de resistência da união ao dente ${ }^{2,4,8}$. De acordo com esta variação, podemos ter bases com diferentes graus de retentividade.

Algumas características como a presença de pontos de solda na base de braquetes metálicos devem ser evitadas para prevenir falhas na vedação marginal do compósito na malha do acessório. O microjateamento da base, assim como a incorporação de partículas metálicas ou cerâmicas, são recursos utilizados que podem resultar em um aumento significante na resistência da colagem, diminuindo as falhas clínicas $5,8,9,19$.

Atualmente, existem vários tipos de braquetes, com diferentes bases, dentre elas podemos citar as de malha fina, malha grossa, as totalmente fundidas ou usinadas e aquelas com sulcos retentivos ${ }^{4,13,20}$.

Smith e Reynolds ${ }^{20}$ avaliaram as diferenças na resistência média dos braquetes, A-Company (malha fina), Dentaurum (malha grossa) e Dynalock (undercut), todos colados com compósito Concise. Os autores concluíram que os braquetes Dentaurum e A-Company tiveram um desempenho melhor que o Dynalock.

Um outro aspecto relevante a ser salientado quanto à forma da base dos braquetes, está relacionado ao grau de penetração do compósito e da luz ativadora ${ }^{8}$. A área da base do braquete também

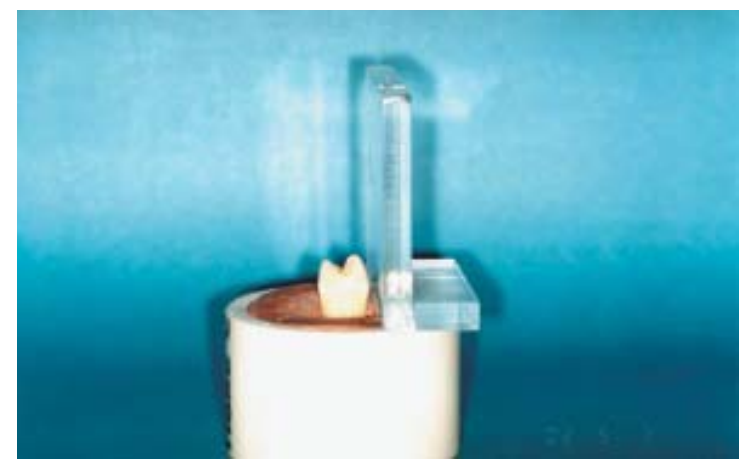

FIGURA 1 - Posicionamento do dente no centro do tubo de PVC, utilizando o posicionador de acrílico. influencia a resistência adesiva, porém nem sempre a base de maior área possui maior retenção. A melhor retenção, na maioria das vezes, está ligada à adaptação da base à superfície dentária ${ }^{5}$.

Baseado nas considerações acima, e com finalidade investigativa, propõe-se neste estudo comparar os braquetes metálicos Monobloc (Morelli), Dynalock (3M Unitek) e Equilibrium (Dentaurum), em relação à resistência ao cisalhamento da colagem e ao ARI (Índice de Remanescente Resinoso).

\section{MATERIAIS E MÉTODOS}

Foram utilizados 36 pré-molares humanos recém extraídos com finalidade ortodôntica, devidamente limpos e armazenados em recipiente plástico contendo solução de formol a 10\%.

Estes dentes foram incluídos em tubos de PVC com gesso pedra tipo IV de maneira centraliza$\mathrm{da}$, posicionando suas faces vestibulares de forma perpendicular à base do troquel, utilizando um posicionador de acrílico em ângulo de $90^{\circ}$ especialmente projetado para este fim (Fig. 1). A seguir, no centro da face vestibular de cada dente foi delimitada uma área correspondente ao tamanho da base do braquete com fita adesiva (Fig. 2).

$\mathrm{Na}$ área previamente preparada foi realizada profilaxia com pedra-pomes e água durante 15 segundos ${ }^{12}$, lavagem abundante também por 15 segundos e em seguida secagem pelo mesmo tempo. Esta região foi submetida ao condicionamen-

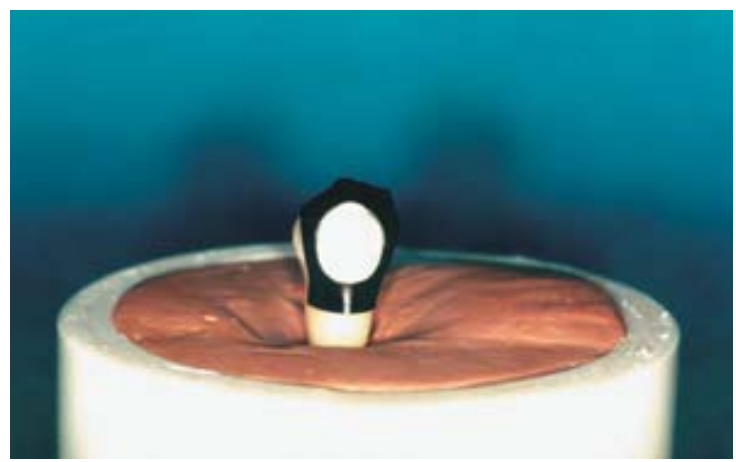

FIGURA 2 - Delimitação de área na superfície vestibular do dente com fita adesiva, previamente à colagem. 


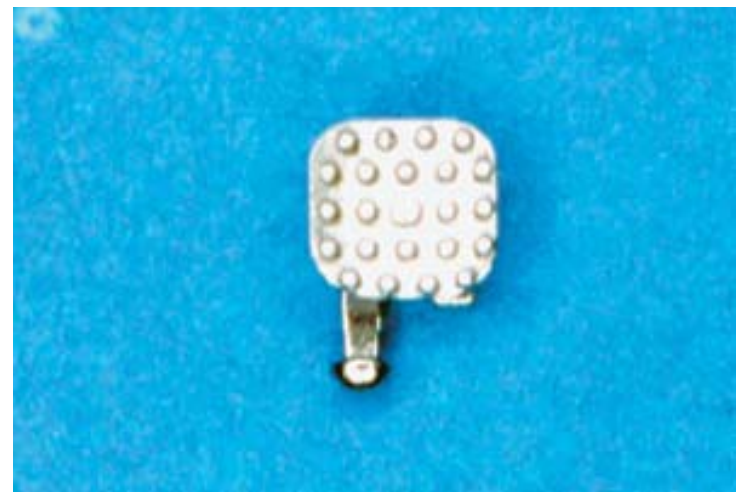

FIGURA 3A - Base do braquete Monobloc.

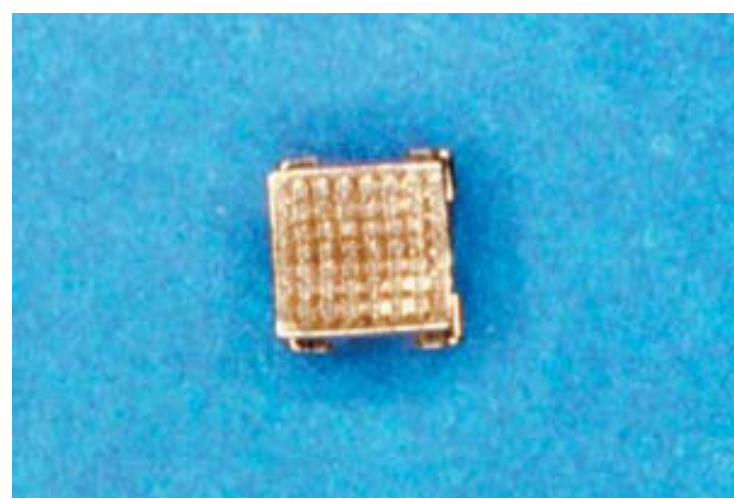

FIGURA 3C - Base do braquete Equilibrium.

to com ácido fosfórico a 37\% (Dentisply) por 20 segundos ${ }^{11}$, lavagem com jato de ar/água por 15 segundos ${ }^{7}$ e secagem pelo mesmo tempo com jato de ar isento de óleo e umidade.

Os 36 pré-molares humanos foram divididos em 3 grupos, sendo cada grupo colado com um tipo de braquete. Foram utilizados os seguintes braquetes: Monobloc (Morelli) - base com pinos retentivos; Dynalock (3M Unitek) - base com sulcos retentivos e Equilibrium (Dentaurum) - base com retenção em forma de malha confeccionada a laser (Fig. 3A, B, C).

Para colagem dos braquetes ao esmalte dentário foi utilizado o compósito Concise Ortodôntico $(3 \mathrm{M})$, segundo as recomendações do fabricante. Todas as colagens foram feitas pelo mesmo operador utilizando uma pinça para colagem (Morelli) tomando o cuidado de pressionar todos os braque-

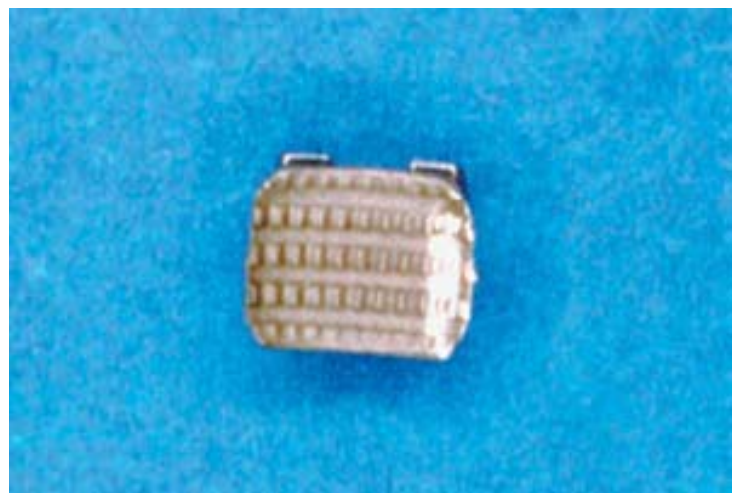

FIGURA 3B - Base do braquete Dynalock.

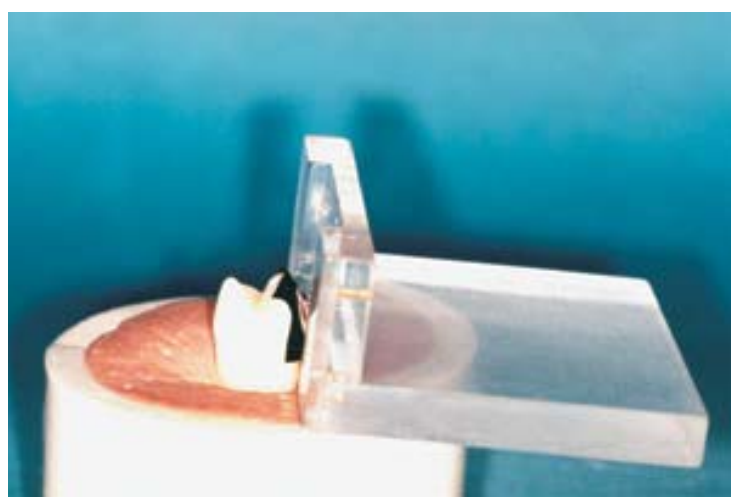

FIGURA 4 - Colagem do braquete com o auxílio do posicionador de acrílico.

tes com a mesma intensidade. $\mathrm{O}$ posicionamento dos braquetes foi avaliado com um posicionador de acrílico e os excessos removidos com o auxílio de uma sonda exploradora (Fig. 4).

Os braquetes foram descolados por uma máquina Universal de Ensaios Mecânicos (EMIC - DL 2000) com uma velocidade de $0,5 \mathrm{~mm}$ por minuto ${ }^{16,18}$ para avaliar a resistência ao cisalhamento da amostra. Os valores de resistência obtidos em Kgf foram então divididos pela área da base de cada tipo de braquete testado, com o objetivo de padronização dos resultados, sendo expressos em $\mathrm{Kgf} / \mathrm{cm}^{2}{ }^{6}$

Após a remoção dos acessórios, cada dente foi classificado de acordo com a quantidade de compósito remanescente no esmalte, usando o ARI (Índice de Remanescente Resinoso), descrito por Artun e Bergland ${ }^{1}$. 


\section{ANÁLISE ESTATÍSTICA}

Os dados obtidos foram protocolados e submetidos ao teste estatístico.

Para avaliação dos dados referentes ao ensaio de cisalhamento, foi utilizada a análise de variância múltipla e o teste complementar de Tukey.

Os resultados do ARI foram submetidos ao teste de $\mathrm{X}^{2}$ (Qui-quadrado de Pearson), para testar a hipótese de que as variáveis são independentes.

\section{RESULTADOS E DISCUSSÃO}

Analisando os dados obtidos pelo Teste de Cisalhamento em relação aos diferentes braquetes empregados, submetidos à análise de variância e teste complementar de Tukey, observou-se diferença significativa, ao nível de $99 \%$ de probabilidade $(\mathrm{p}<0,01)$ em relação ao Teste de Cisalhamento. O braquete Monobloc ( $\mathrm{x}=28,19$ $\pm 7,90$ ) apresentou maior valor médio quando comparado aos braquetes Dynalock ( $\mathrm{x}=18,24$ $\pm 5,89$ ) e Equilibrium ( $x=18,07 \pm 7,03)$. Estes dados podem ser observados no gráfico 1 .

Os escores do ARI de cada grupo foram analisados estatisticamente pelo teste $\mathrm{X}^{2}$ (Qui-Quadrado de Pearson) (Gráf. 2). O grupo de braquetes Dynalock obteve o maior valor médio $(\mathrm{x}=1,33)$, seguido pelo grupo de braquetes Monobloc $(\mathrm{x}=0,80)$ e Equilibrium $(\mathrm{x}=0,73)$.

$\mathrm{O}$ valor de resistência adesiva de um material para suportar os esforços clínicos deve estar entre 60 a $80 \mathrm{Kgf} / \mathrm{cm}^{2}$, segundo Reynolds e Fraunhofer ${ }^{15}$. Em outro trabalho, Beltrami et al. ${ }^{3}$ encon-

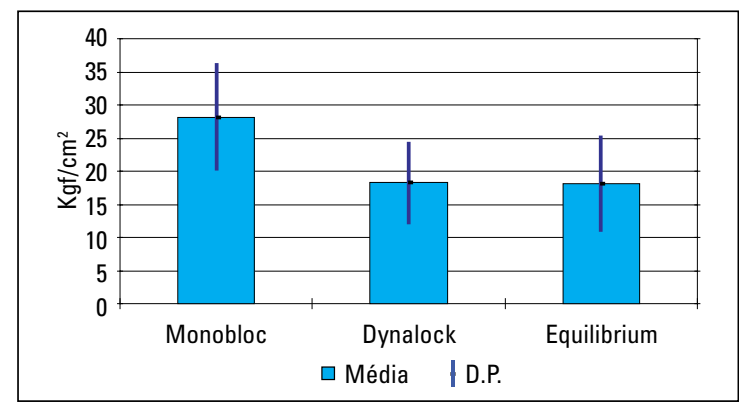

GRÁFICO 1 - Médias e desvios-padrões das medidas de resistência ao cisalhamento. traram valores de resistência ao cisalhamento para braquetes Unitek 377 e Dentaurum, ambos com a base em tela, de aproximadamente de $48,4 \mathrm{Kgf} / \mathrm{cm}^{2}$ e $81,6 \mathrm{Kgf} / \mathrm{cm}^{2}$, respectivamente.

Neste estudo encontramos valores inferiores aos relatados pelos autores acima nos três grupos testados (Monobloc x = 28,19; Dynalock $\mathrm{x}=18,24$; Equilibrium $x=18,07)$. Esta diferença provavelmente se deve ao fato de que neste experimento foram utilizados pré-molares humanos que apresentam alta porcentagem de esmalte aprismático ${ }^{17}$ e também a diferenças de metodologia e materiais empregados.

O tipo de adesivo e suas características influenciam diretamente na adesão, assim, utilizou-se neste estudo o Concise Ortodôntico para obter uma padronização dos grupos de forma que os valores encontrados fossem a expressão das diferenças na retenção dos diferentes tipos de bases estudadas.

O tipo de base do braquete interfere diretamente na resistência ao cisalhamento da colagem $^{4,8}$. Os braquetes Monobloc possuem uma base com retenção em forma de pinos, os braquetes Dynalock apresentam sua base com sulcos retentivos e os braquetes Equilibrium possuem a base em malha com retenção confeccionada a laser. As diferenças de resistência de acordo com o tipo de retenção da base foram evidenciadas neste trabalho, visto que o grupo formado por braquetes Monobloc foi estatisticamente superior aos grupos dos braquetes

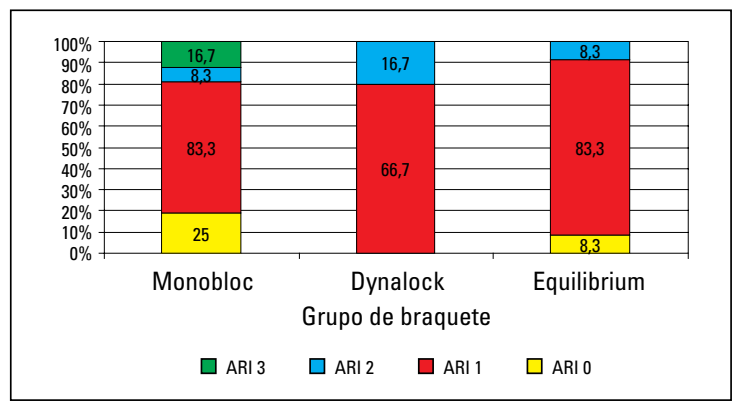

GRÁFICO 2 - Distribuição do Índice de Remanescente Resinoso (ARI). 
Dynalock e Equilibrium. Entre estes dois últimos grupos não foram encontradas diferenças estatísticas significantes, apesar da retenção de suas bases serem totalmente diferentes.

Divergindo deste estudo, Beltrami et al. ${ }^{3}$ relataram que um braquete experimental com sulcos retentivos na base apresentou superioridade estatística em relação aos braquetes Dentaurum 705-0225, Unitek 019-321, Unitek 019-312/377, Morelli ${ }^{\circ} 15$, entre outros.

A avaliação da superfície dentária após descolagem dos acessórios é de vital importância para verificar o remanescente de material de colagem em diferentes tipos de braquetes, para isto foi utilizado os escores de Artun e Bergland ${ }^{1}$. Assim, não foi encontrada diferença estatística significante entre os grupos da amostra em relação ao ARI (Índice de Remanescente Resinoso).

Nesta avaliação, constatamos que na maioria dos corpos de prova, menos da metade do compósito utilizado na colagem ficou aderido ao esmalte. Diante deste fato pode não ter ocorrido uma adequada adesão ao esmalte e/ou pode ter havido durante a descolagem fraturas na estrutura do esmalte. Esta segunda hipótese poderá ser confirmada somente com análise microscópica do esmalte dos dentes da amostra.

\section{CONCLUSÕES}

Em concordância com os resultados obtidos, podemos concluir que:

1) O grupo formado por braquetes Monobloc foi estatisticamente superior aos grupos dos braquetes Dynalock e Equilibrium em relação à resistência ao cisalhamento da colagem;

2) Não houve diferença estatística significante entre os grupos com braquetes Dynalock e Equilibrium em relação à resistência ao cisalhamento da colagem;

3) $\mathrm{Na}$ avaliação do ARI, não houve diferença estatística significante entre os grupos testados;

4) Durante o processo de descolagem o maior número de fraturas ocorreu entre o esmalte e o braquete nos três grupos estudados.

\title{
Analysis of the adhesion quality of different metallic bracket bases
}

\begin{abstract}
Considering the differences in the bracket bases currently used, it was performed a study with the purpose of comparing three different metallic bracket bases commercially available. It was used 36 human bicuspids divided into 3 groups of 12 teeth. These teeth were immersed in a troquel with gypsy stone type IV and positioned with their buccal face perpendicular to the troquel base. For each group of teeth it was bonded one of the three brackets using Concise Orthodontic composite. Afterwards they were submitted to a shear bound strength test in a universal machine calibrated for a $0,5 \mathrm{~mm} /$ minute speed. Monobloc bracket base showed the greater mean value of adhesive resistance $\left(x=28,19 \mathrm{Kgf} / \mathrm{cm}^{2}\right)$, result statistically superior to that shown by Equilibrium $\left(x=18,07 \mathrm{Kgf} / \mathrm{cm}^{2}\right)$ and Dynalock $\left(x=18,24 \mathrm{Kgf} / \mathrm{cm}^{2}\right)$ ones. The ARI (Adhesive reminiscent index) showed no statistically difference among the bracket bases studied.
\end{abstract}

Key words: Orthodontic. Bonding. Shear bond strength. Bracket. Base. 


\section{REFERÊNCIAS}

1. ARTUN, J.; BERGLAND, S. Clinical trials with crystal growth conditioning as an alternative to acid-etch enamel pretreatment. Am J Orthod, St. Louis, v. 85, no. 4, p. 333 - 340, Apr. 1984.

2. ASSIS, R. D.; JANSEN, W. C.; MOTA, J. M. L. F. Influência do microjateamento na retentividade de bráquetes metálicos de fabricação nacional. Arquivos em Odontologia, Belo Horizonte, v. 35, n. $1 / 2$, p. $71-83$, jan./dez. 1999

3. BELTRAMI, L. E. R. et al. Desenvolvimento de um bráquete para colagem direta de sulcos retentivos na base e sua comparação com os similares com tela. Ortodontia, São Paulo, v. 17, n. 3, p. 5 - 14, jan./dez. 1984.

4. BELTRAMI, L. E. R.; FREITAS, C. A.; MARTINS, D. R. Bráquetes com sulcos retentivos na base, colados clinicamente e removidos em laboratório por testes de tração, cisalhamento e torção. Ortodontia, São Paulo, v. 29, n. 2, p. 27- 39, maio/ago. 1996.

5. FLORIANO, $\mathrm{H}$. et al. Estudos da resistência à tração de braquetes metálicos colados em relação a alguns tipos de base. Rev Paul Odontol, São Paulo, v. 23, n. 2, p. 20 - 23, mar./abr. 2001.

6. GANDINI, M. R. E. A. S. et al. Bráquetes para colagem direta: estudo das características referentes à base de fixação. Ortodontia, São Paulo, v. 22, n. 1, p. 5 -13, jan./abr. 1989.

7. GWINNETT, A. J. Acid etching for composite resins. Dent Clin North Am, Philadelphia, v. 25, no. 2, p. $271-289$, Apr. 1981

8. KNOX, J. et al. The influence of bracket base design on the strength of the bracket-cement interface. Eur J Orthod, London, v. 27, no. 3, p. $249-254$, Sept. 2000.

9. MAIJER, R.; SMITH, D. C. Variables influencing the bond strength of metal orthodontic bracket bases. Am J Orthod, St. Louis, v. 79 , no. 1 , p. $20-34$, Jan. 1981

10. MAIJER, R.; SMITH, D. C. Improvements in bracket base design. Am J Orthod, St. Louis, v. 83, no. 4, p. $277-281$, Apr. 1983

11. MARDAGA, W. J.; SHANNON, I. L. Decreasing depth of etch for direct bonding in orthodontics. J Clin Orthod, Boulder, v. 16 no. 2, p. $130-132$, Feb. 1982.
12. PUS, M. D.; WAY, D. C. Enamel loss due to orthodontic bonding with filled and unfilled resins using various clean-up techniques. Am J Orthod, St. Louis, v. 77, no. 3, p. 269 - 283, Mar. 1980

13. REGAN, D.; VAN NOORT, R. Bond strengths of two integral bracket-base combinations: an in vitro comparison with foil-mesh. Eur J Orthod, London, v. 11, no. 2, p. 144 - 153, May 1989.

14. RETIEF, D. H.; SADOWSKY, P. L. Clinical experience with the acid- etch technique in orthodontic. Am J Orthod, St. Louis, v. 68 , no. 6, p. $645-654$, Dec. 1975.

15. REYNOLDS, I. R.; FRAUNHOFER, J. A. Direct bonding in orthodontics: A comparison of attachments. Br J Orthod, London, v. 4 , no. 2 , p. $65-69,1976$.

16. RICHARD, G. M. Condicionamento ácido do esmalte dentário: efeito do tempo sobre a resistência ao cisalhamento e modos de fratura da colagem. 1993. Dissertação (Mestrado em Ortodontia) - Faculdade de Odontologia, Universidade Federal do Rio de Janeiro, 1993

17. RIPA, L. W.; GWINNETT, A. J.; BUONOCORE, M. G. The "prismless" outer layer of decidous and permanent enamel. Arch Oral Biol, Oxford, v. 11, no. 1, p. 41 - 48, Jan. 1996.

18. ROMANO, F. L. Estudo comparativo da resistência ao cisalhamento da colagem e do Índice de Remanescente Resinoso (ARI) entre os compósitos concise e superbond: estudo "in vitro". 2001. 100 f. Trabalho de Conclusão de Curso (Especialização em Ortodontia e Ortopedia Facial) - Escola de Farmácia e Odontologia de Alfenas, Alfenas, 2001

19. PINTO, Ary dos Santos et al. A reciclagem de bráquetes na clínica ortodôntica. Ortodontia, São Paulo, v. 29, n. 2, p. 63-67, maio/ago. 1996.

20. SMITH, N. R.; REYNOLDS, I. R. A comparison of three bracket bases: an in vitro study. Br J Orthod, London, v. 18, no.1, p. 29 - 35, Feb. 1991.

21. THANOS, C. E.; MUNHOLLAND, T.: CAPUTO, A. A. Adhesion of mesh-base direct-bonding brackets. Am J Orthod, St. Louis, v. 75 , no. 4 , p. $421-430$, Apr. 1979.
Endereço para correspondência

Danielle Morello Park

Rua Campos Sales, 1191 Apto. 92 - Higienópolis

Ribeirão Preto - SP

CEP: 14015-110

E-mail: danipark@directnet.com.br 